\title{
DIDACMER \\ Didacticiel et encyclopédie de Construction à la mer
}

\author{
Ch. Brossard, Ingénieur en Chef des Ponts et Chaussées (E.R.)
}

A. Grovel, Professeur à l'Université de Nantes et Responsable scientifique du projet DIDACMER

P. Quinaou, Responsable du projet DIDACMER

\section{Introduction}

a) Une tradition, depuis l'Amirauté de l'Ancien Régime, confie aux Ingénieurs du Corps des Ponts et Chaussées la responsabilité de la gestion du domaine maritime littoral, ainsi que la construction et l'exploitation des ports maritimes autres que ceux d'intérêt militaire.

C'est tout naturellement donc, qu'une formation technique spécialisée s'est développée, au bénéfice des divers groupes d'ingénieurs et de techniciens, pour atteindre au niveau de compétence requis par ces activités du génie civil.

Sur le vaste champ d'application du littoral de l'Hexagone et des pays de l'Outre-Mer, très diversifiés par les données naturelles de leurs sites côtiers, l'observation, les études et les réalisations ont forgé le "savoir-faire » d'une véritable école française des Travaux maritimes.

Les signatures des cours spécialisés qui ont traité la matière durant les 50 dernières années témoignent de la richesse et de la qualité pédagogiques de leurs auteurs : MM. Laval, Blosset, larras, Chapon, Graillot.

Mais après une longue période d'aménagements exemplaires qui ont placé notre pays à un niveau d'équipement envié, le domaine d'intérêt des ports s'est légitimement réorienté au profit d'une exploitation plus efficace et de mesures de marketing commercial de nature à tirer le meilleur profit économique des équipements disponibles.

On a dès lors assisté à une réduction d'intérêt pour cette discipline technique des travaux à la mer, qui a pu mettre en question le maintien des modules de formation dans les programmes d'enseignement de nos Grandes Ecoles. b) Ce domaine n'est donc pas épargné par la remise en cause permanente de la pédagogie des divers secteurs de l'enseignement.

Le constat des besoins de la formation et de la pratique professionnelle du secteur technique Travaux publics, a dégagé les lignes directrices d'un renouveau de la formation :

- l'ingénieur est formé, en phase initiale, à une polyvalence d'application de ses techniques de base. Il " apprend à apprendre " et s'adaptera ensuite au cours de sa vie professionnelle, à la satisfaction de la demande du marché ; - si l'ingénieur d'études, maintient le contact avec les disciplines fondamentales du métier, l'ingénieur travaux a par contre un champ d'action d'Organisateur - Gestionnaire - Réalisateur, dont la pratique quotidienne érode trop rapidement le savoir théorique et ne permet pas d'actualiser la connaissance dont la rapidité d'évolution est connue ;

- le Chantier, centre d'activités très décentralisé, est un milieu d'isolement - souvent géographiquement très dispersé - dont le rythme de décision impose le temps réel. Le responsable doit être soutenu par des aides à la décision : recyclage court, sur des thèmes pratiques, dont la mise à jour fréquente garantit l'actualité technique.

c) Formation alternée, Recyclage, Formation continue, toutes les filières d'adaptation des domaines de l'enseignement, sont à développer, en associant leur complémentarité. 


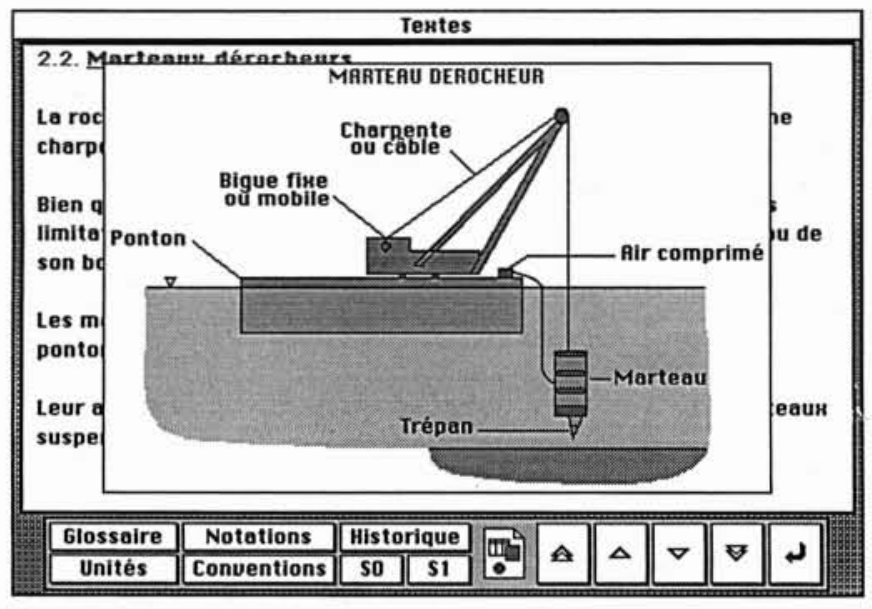

1. Extrait du chapitre "Déroctage ".

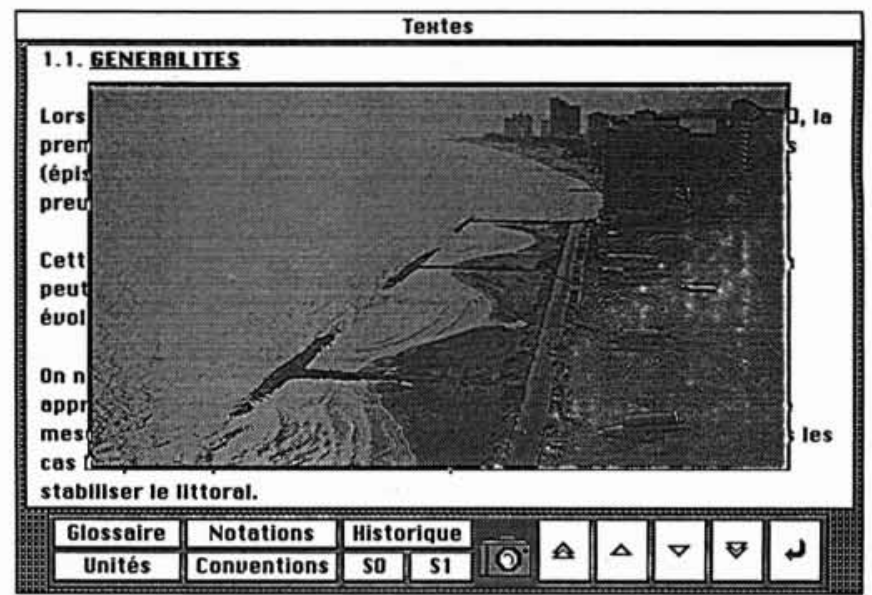

2. Extrait du chapitre "Ouvrages de défense des côtes».
"DIDACMER " outil pédagogique d'aujourd'hui, présenté ci-après, possède de nombreux caractères d'intérêt répondant à cette nécessaire évolution vers un meilleur service à l'Ingénieur maritime.

Le matériel micro-informatique de large diffusion va développer très rapidement la technique multimedia, ainsi que l'ensemble des experts s'en déclarent d'accord. Dans les positions de l'Ingénieur précédemment évoquées, il offrira le véritable cours particulier à domicile, adapté à sa situation imposée d'autodidacte dans le domaine spécifique de son problème du moment. Les garanties du succès sont réunies :

- par document unique, il accède aux divers modules des disciplines de base qui commandent sa technique spécialisée : Océanologie, Géotechnique, Mécanique et Calcul de structure, Hydraulique. Les choix ont été effectués par le collège d'experts, et l'utilisateur n'a pas à s'assurer par lui-même de l'excellence des sources qui lui sont proposées ;

- la mise à jour en est assurée à la fréquence requise. Globalement sont réintroduites les références les plus récentes d'ouvrages réalisés et le bénéfice d'expérimenta- tion partagée est mis à disposition du plus grand nombre ; - la création d'un " club d'utilisateurs " apporte la garantie de l'adaptation aux attentes du praticien. Cette interactivité en temps différé est essentielle à la satisfaction des attentes d'efficacité et de qualité du produit.

Nous entrons dès maintenant, de plain-pied dans ce plus vaste projet d'une université ouverte, où l'acquisition et le partage de la connaissance n'exigeront plus la présence physique dans le lieu et dans l'instant où devaient jusqu'alors se réunir professeurs et professionnels concernés par la discipline spécialisée des travaux maritimes. A son rythme propre, et en fonction de son histoire technique personnelle, l'utilisateur appellera la ressource pédagogique utile au projet qu'il poursuit.

Songeons un instant à tous nos collègues Ingénieurs francophones aujourd'hui dispersés de par le Monde. Ils ont bénéficié, durant les durées variables, de notre enseignement auquel ils demeurent attachés : est-il un meilleur moyen à leur offrir pour maintenir la chaîne, et leur permettre ainsi la perception pertinente des projets de nos sociétés d'ingénierie et de nos entreprises?

\section{Présentation de DIDACMER}

Le but de DIDACMER est double :

- l'autoformation pour les cadres et ingénieurs de Génie civil aux techniques particulières des travaux maritimes. Le niveau minimum requis est celui d'un DUT Génie civil. Un diplôme d'Université de Travaux maritimes, délivré par l'Université de Nantes, peut ponctuer cette formation;
- la consultation dans un bureau d'étude ou une entreprise pour des projeteurs, mais aussi des spécialistes des méthodes, des commerciaux et des décideurs.

Pour cela DIDACMER regroupe plus de 10000 pages, 4000 schémas, 500 photographies, 2500 formules articulés selon un plan commun aux trois modes: cours (300 heures), exercices, encyclopédie. Au total la somme des données sur le disque atteint 300 méga-octets. 


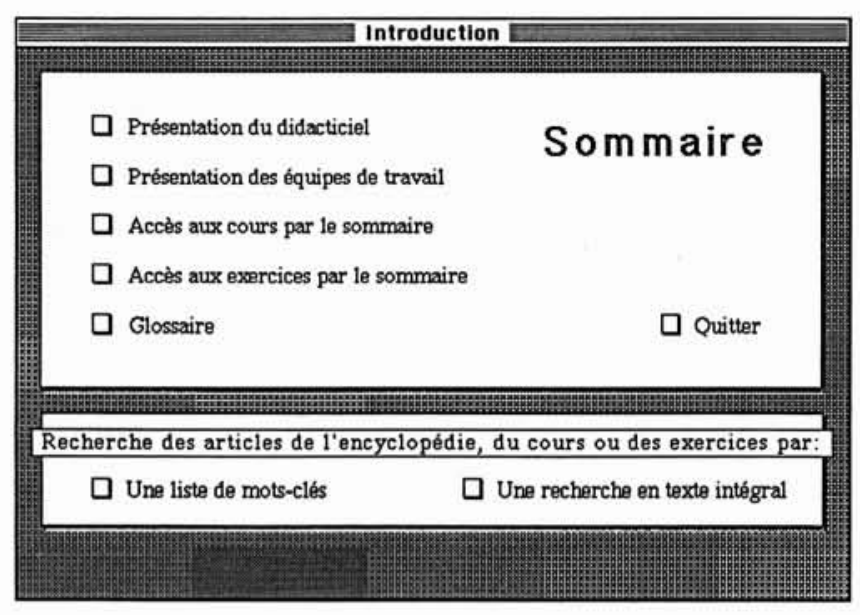

3. Sommaire général.

\begin{tabular}{|c|c|c|c|c|c|c|c|c|}
\hline \multicolumn{9}{|c|}{ 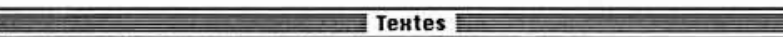 } \\
\hline \multicolumn{9}{|c|}{ 1.2. LES AMORTISSEURS } \\
\hline \multicolumn{9}{|c|}{ 1.2.1. Généralités - Rôle } \\
\hline \multicolumn{9}{|c|}{$\begin{array}{l}\text { Entre la coque du navire et le duc d'albe, l'on insère une structure } \\
\text { secondaire que l'on appelait autrefois défense et que l'on nomme } \\
\text { actuellement "SYSTEME AMORTISSEUR SECONDRIRE" ou "RMORTISSEUR" } . \text {. }\end{array}$} \\
\hline \multicolumn{9}{|c|}{$\begin{array}{l}\text { Le rôle de l'amortisseur est de participer à l'absorption d'énergie de } \\
\text { l'accostage ou de l'amarrage, car ll est capable d'une grande } \\
\text { déformation. II répond en cela au critère de déformabilité auquel doit } \\
\text { satisfaire le đuc đ'Albe. }\end{array}$} \\
\hline \multicolumn{9}{|c|}{ Уоісі l'eкеmple de la déformation d'un amortisseur : } \\
\hline Glossaire & Notations & His & ique & & & & & \\
\hline Unités & Conventions & $\overline{\text { SO }}$ & S1 & $\Delta$ & $\Delta$ & $\nabla$ & $\nabla$ & لم \\
\hline
\end{tabular}

4. Ecran courant.

\subsection{Les thèmes abordés dans DIDACMER}

Le plan selon lequel s'organisent les cours, exercices et encyclopédie de DIDACMER, et dont le détail est présenté dans la notice du didacticiel, comporte deux parties distinctes :

\section{A. Rappel des notions théoriques.}

A.1. Culture générale scientifique

Mathématiques, Résistance des matériaux, Structures, Mécanique des sols et des roches, Géophysique, Hydraulique des sols, Mécanique rationnelle et vibratoire.

\section{A.2. Hydraulique maritime}

Météorologie marine, Courants, Marée, Houle, Théorie des modèles.

\section{A.3. Océanologie}

Géodésie maritime, Géotechnique marine, Océanographie dynamique, Fonds marins et vie, Régime des côtes.

\section{B. Ingénierie des travaux maritimes proprement dite.}

\section{B.1. Paramètres de conception des ouvrages}

Navire et géométrie portuaire, Etudes préliminaires et stratégie de construction, Fonctions portuaires.

\section{B.2. Technologie de construction}

Revue des méthodes générales de construction, Matériels adaptés aux travaux maritimes.

\section{B.3. Matériaux}

Enrochements, Bétons hydrauliques, Aciers, Bois, Matériaux divers.

\section{B.4. Ouvrages d'accès}

Chenaux d'accès, Dragage, Déroctage, Systèmes d'aide à la navigation.

\section{B.5. Ouvrages de protection}

Digues et brise-lames, Ouvrages de défense des côtes.

B.6. Ouvrages fonctionnels

Quais continus, Ouvrages sur pieux, Formes, Bassins, Ecluses.

\subsection{Les fonctionnalités de DIDACMER}

DIDACMER fonctionne entièrement à l'aide de la souris et seule la réponse à certaines questions et l'écriture de mots-clés requièrent une intervention au clavier de l'utilisateur.

Le sommaire général (fig. 3)

Il donne accès :

- aux séquences de présentation de DIDACMER et à l'explication des différentes fonctions,

- aux séquences de présentation des partenaires et collaborateurs de DIDACMER,

- aux cours de DIDACMER,

- aux exercices,

- au glossaire,

- aux deux modes de recherches de DIDACMER (par mots-clés ou en texte intégral).

Pour cela il suffit d'activer, à l'aide de la souris, le carré de renvoi $\square$ précédant le mode souhaité.

La présentation des données et les fonctionnalités courantes

Un écran courant (fig. 4) présente, qu'il s'agisse du cours, d'un exercice ou d'un article encyclopédique, les mêmes caractéristiques. On y trouve :

- un hypertexte qui peut être illustré de schémas ou de photographies et permet (par le biais de carrés de renvoi) un accès à une illustration supplémentaire (photographie, schéma ou animation graphique) ou à un complément sous forme textuelle;

- une barre de fonctionnalités permettant:

- le voyage dans le texte grâce aux touches d'avance ou de recul page par page ou ligne par ligne,

- un accès à l'écran précédant grâce à la touche retour, - un accès au glossaire, 


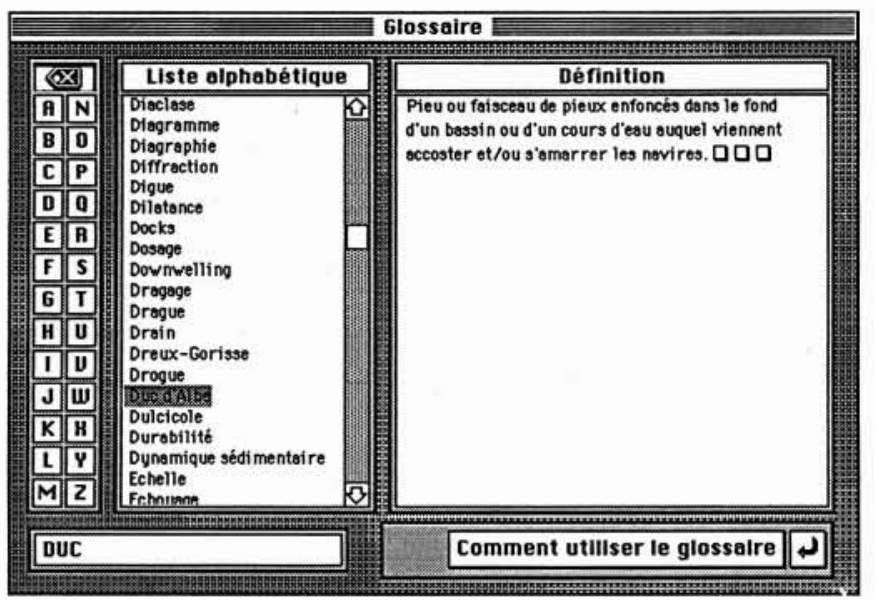

5. Glossaire.

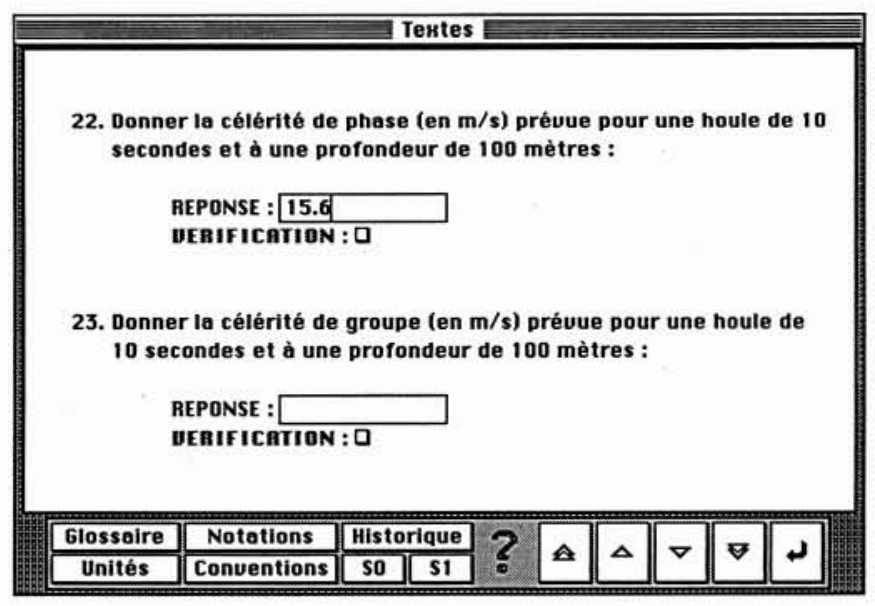

6. Répondre à un exercice.

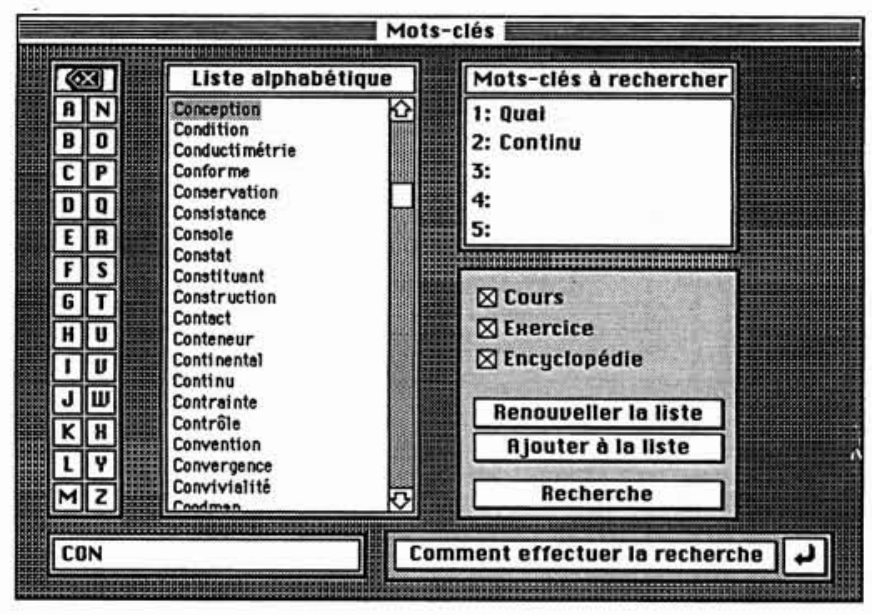

7. Recherche par mots prédéfinis.

8. Résultat d'une recherche d'articles.

- un accès à l'historique qui mémorise les dix dernières étapes parcourues par l'utilisateur,

- un accès au sommaire du mode où l'on se situe (S1),

- un accès au sommaire général (S0).

Le glossaire (fig. 5)

Le glossaire définit environ 500 mots dont plus de la moitié sont illustrés. Pour choisir un mot il suffit de le rechercher à l'aide de l'ascenseur ou bien du tableau alphabétique en y inscrivant les premières lettres du mot en question. Puis, il faut cliquer sur ce mot pour faire apparaître sa définition.

Les exercices (fig. 6)

DIDACMER propose pour chaque chapitre du cours une série de questions ou d'exercices. Il s'agit de questions/réponses simples ou bien de questions à choix multiples ou encore d'applications numériques seules nécessitant une réponse au clavier de l'utilisateur. Selon la réponse,
DIDACMER oriente l'utilisateur vers la solution ou des compléments d'information.

\section{Les modes de recherche}

\section{Recherche par mots-clés prédéfinis (fig. 7)}

Ce mode utilise une liste de mots-clés prédéfinis. Ces mots-clés orientent l'utilisateur vers des textes présélectionnés. On peut ainsi choisir jusqu'à cinq mots-clés. Le résultat de la recherche présente la liste des articles correspondant à l'intersection des listes d'articles prédéfinies affectées à chaque mot-clé.

\section{Recherche en texte intégral (fig. 9)}

Ce mode permet d'inscrire jusqu'à cinq mots-clés que l'utilisateur inscrira lui-même dans la liste des mots à rechercher. Le résultat de la recherche fait apparaître la liste des articles correspondant à l'intersection des listes d'articles où se trouve chaque mot-clé. 


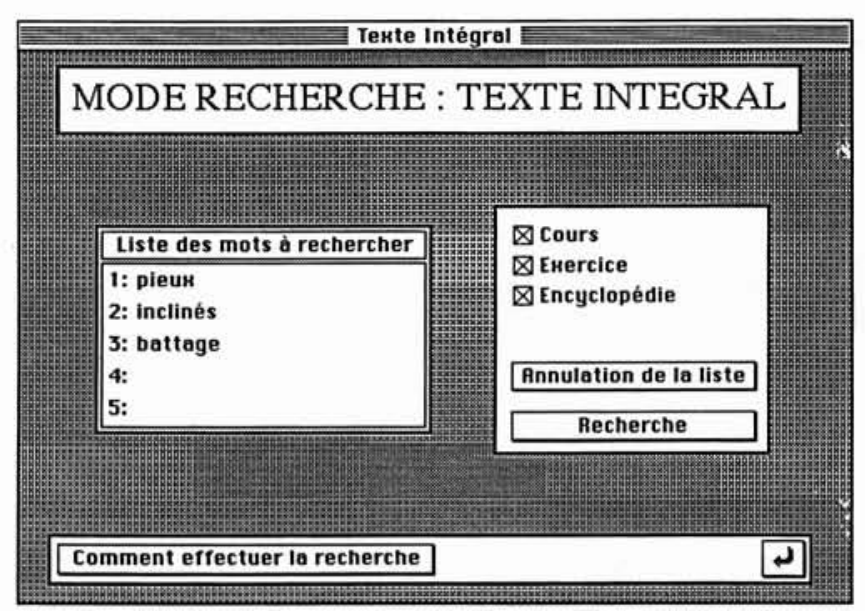

9. Recherche en texte intégral.

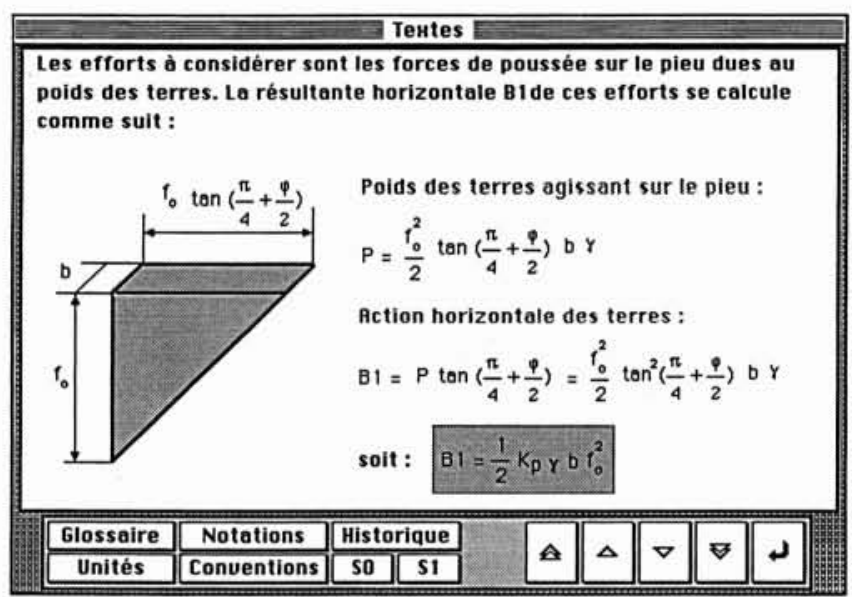

10. Extrait de "Calcul d'un Duc d'Albe ».

\section{Comment utiliser DIDACMER dans la pédagogie ?}

En formation initiale une première expérimentation a déjà eu lieu dans le DEA de Génie civil du Grand Ouest, qui regroupe des Ingénieurs et des MST de Génie civil des Universités de Nantes, Le Havre, Caen et d'Ecoles d'Ingénieurs, ECN et INSA de Rennes.

Ce DEA comporte une option Génie côtier avec un enseignement faisant le point des connaissances en Travaux maritimes pour de jeunes ingénieurs désirant, avant un travail de recherche, parfaire leurs connaissances en Travaux maritimes.

La consultation sur DIDACMER leur a d'abord été présentée, puis les formateurs ont laissé les microordinateurs équipés de lecteurs CD-ROM en libre service avec comme support d'interrogation un avant projet sommaire de station d'éclatement pétrolière.
Une fiche permettant de suivre le fil d'Ariane de l'utilisateur est donnée à chacun. Elle doit être remplie par suites de mots-clés.

L'expérience a prouvé que l'assiduité était très grande. Les étudiants ont utilisé DIDACMER : comme une encyclopédie, comme une aide à la rédaction de projets par l'examen des études de cas, ou comme une aide à la rédaction de notes de calculs. Plus, certains s'en sont servis dans d'autres domaines comme un support de révision de connaissance. Il est vrai qu'il s'agissait là d'un groupe d'utilisateurs particulièrement doués et motivés.

Cette expérience a conduit les formateurs à envisager un deuxième mode d'utilisation en pédagogie, destiné celui-là à un niveau légèrement inférieur, les étudiants de $2^{\mathrm{e}}$ année (i.e. des Bac +4 ) de la maîtrise de Sciences et techniques de travaux publics et maritimes. Ces étudiants se destinent à un emploi d'Ingénieurs-Travaux oủ ils sont déjà particulièrement apréciés.

Le montage projeté s'apparente à celui qui est utilisé en consultation bibliographique à la Bibliothèque Sainte Geneviève de Paris. Un micro-ordinateur équipé d'une carte spéciale, pilote 20 serveurs avec écran et souris. Vingt étudiants peuvent donc, à leur vitesse et suivant des arbres de consultation personnels, consulter simultanément un seul CD-ROM DIDACMER. L'enseignant est alors un guide, un véritable formateur et non plus uniquement un répétiteur de connaissances.

Ce montage est en cours de réalisation et le Service Formation continue de l'Université de Nantes pourra donc conseiller tous les organismes intérieurs par ce mode d'enseignement.

En troisième lieu, le service de suivi DIDACMER a reçu quelques confidences d'utilisateurs en auto-formation. Il n'y a jamais de remarques informatiques; l'utilisation est donc considérée comme très simple. Par contre, il semble qu'il y ait parfois quelques erreurs d'introduction de motsclés. Il faut donc rappeler que la consultation du menu général (sommaire) et du répertoire général des mots-clés simplifie le travail de recherche, (comme dans toute bibliothèque-papier).

\section{La réalisation informatique}

Les petits disques brillants, multicolores que sont les CDROM sont si fascinants que l'on ne réalise pas vraiment que ces petites merveilles ne constituent que des supports de l'information. Contrairement au livre, le disque ne peut transmettre l'information directement à l'utilisateur, ce qui exige qu'il soit utilisé au sein d'un système d'information.

Ce système d'information, dans le cas de DIDACMER est basé sur deux logiciels dédiés (l'un spécifique à l'environnement Macintosh, l'autre spécifique à l'environnement IBM-PS ou AT-) qui permettent l'accès rapide aux informations stockées sur le disque et qui supportent une couche interface utilisateur de type HyperTexte. 


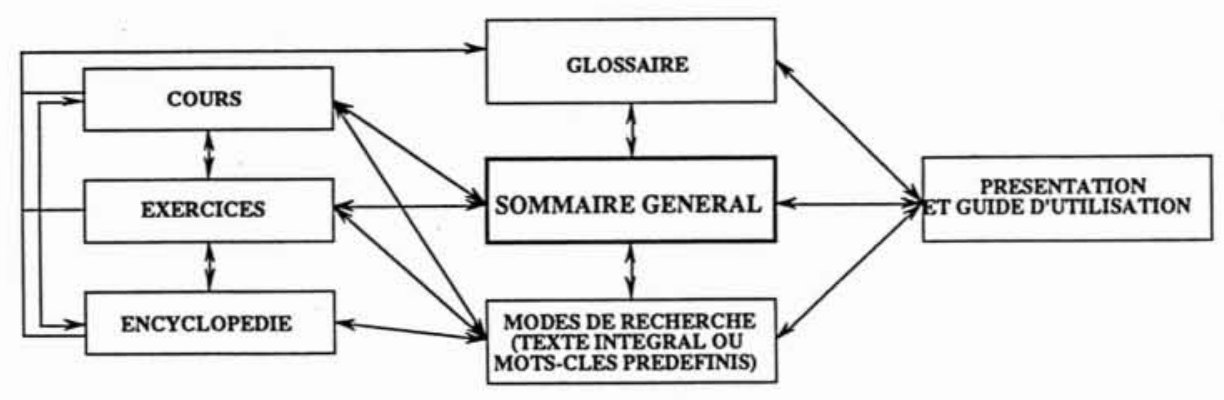

11. Cheminements entre les différents modes de DIDACMER.

Ces logiciels ont nécessité, en amont, une organisation très structurée de la saisie des données et la réalisation d'un logiciel de formatage et d'indexation de cette base.

L'écriture des logiciels de DIDACMER et le prémastering du CD-ROM sur CD-PUBLISHER a été confiée au Bureau van Dijk, un des premiers spécialiste européens du support CD-ROM et réalisateurs de CD-ROM tels que le Robert Electronique, le Kompass France sur CD, Electre, CD-REEF, le CD-ROM VIDAL. Rhône-Poulenc SDS I, II et III... La saisie des données a été entièrement réalisée à l'Université de Nantes au Centre Multimédia du Service Formation Continue.

\subsection{La structure du disque CD-ROM}

Le format physique du CD-ROM a été conçu par Philips et Sony les développeurs de la technologie du disque compact ; ce format est décrit dans un document connu sous le nom de "Yellow Book".

La surface d'un CD-ROM, observée à l'aide d'un microscope, se présente comme une spirale de microcuvettes de la taille d'un micron; le rayon laser et les circuits électroniques implantés sur le lecteur convertissent ces micro-cuvettes en données binaires qui, une fois traitées, sont envoyées à l'ordinateur secteur par secteur. Chaque secteur contient 2352 octets d'informations, dont 2048 réservés à l'utilisateur, l'ensemble étant subdivisé de la manière suivante :

$\begin{array}{ll}\text { - synchronisation } & 12 \text { octets } \\ \text { - en-tête } & 4 \text { octets } \\ \text { - données utilisables } & 2048 \text { octets } \\ \text { - code détecteur d'erreur (CDE) } & 4 \text { octets } \\ \text { - espace inutilisé } & 8 \text { octets } \\ \text { - code correcteur d'erreur (CCE) } & 276 \text { octets }\end{array}$

\section{Synchronisation}

Les secteurs ne sont pas physiquement séparés : le début de chaque secteur est simplement repéré par une séquence de 12 octets appelé code de synchronisation. Le lecteur de CD-ROM suit la position de la tête laser sur le disque en repérant le nombre d'octets qui défilent devant celle-ci et en s'appuyant sur les informations délivrées par le code de synchronisation. Des méthodes d'avertissement et de repé- rage sont également mises en œuvre car les octets de synchronisation seuls ne suffisent pas à établir de manière bi-univoque la frontière entre les secteurs, la même séquence d'octets de synchronisation pouvant, en effet, se retrouver dans les données utilisateurs.

\section{En-tête}

Les 4 octets de l'en-tête identifient le secteur. Les 3 premiers octets contiennent l'adresse du secteur qui permettra au drive de localiser un secteur particulier. Le dernier octet de la séquence est appelé octet de mode et donne simplement une information de présence du code correcteur d'erreur: le mode 1 inclut une correction d'erreur et une détection d'erreur; le mode 2 n'inclut pas les 280 octets de codes détecteur et correcteur d'erreur et peut-être utilisé pour stocker d'autres informations.

\section{Données utilisateur}

Il s'agit de l'endroit où l'information utilisable est stockée, qu'il s'agisse de texte, de son, de vidéo, etc... Le processus de prémastering consiste à découper les fichiers de données en blocs de 2048 octets, à calculer les codes correcteur et détecteur d'erreur et à ajouter les informations de synchronisation et d'en-tête.

\section{Code détecteur d'erreur (CDE)}

Le code détecteur d'erreur est une valeur de 16 bits générée à partir des valeurs de synchronisation, de l'en-tête et des données utilisateur que le lecteur utilise au travers d'un algorithme pour déterminer la présence d'une erreur apparue au niveau de la lecture. Le CDE est calculé lors de la phase de prémastering.

Espace non utilisé

Cet espace n'est pas utilisé par le CD-ROM, mais a été déplacé et redéfini dans le standard CD-I.

\section{Code correcteur d'erreur (CCE)}

Les octets du code correcteur d'erreur sont utilisés à corriger les éventuelles erreurs de lecteur lorsqu'elles ont été détectées. Un algorithme sophistiqué basé sur un code Reed-Solomon est utilisé.

La densité d'information sur un disque compact est considérable et approche les 100 millions de bits au centimètre carré; avec des densités aussi élevées, même un défaut microscopique peut être la source de centaines d'erreurs. 


\subsection{Les logiciels}

Les logiciels d'accès aux informations et de présentation des données utilisent des logiciels de base écrits par le Bureau van Dijk, mais sont développés dans leurs couches hautes pour répondre spécifiquement à l'application telle qu'elle a été décrite précédemment et qui correspond au cahier des charges de l'Université de Nantes.

Ces logiciels permettent de voyager dans la base selon le schéma suivant (fig. 11):

Pour la recherche des informations et leur présentation selon certaines normes, ces logiciels interprètent des tables créées par un logiciel d'indexation et de formatage à partir des données structurées, au moment de la saisie, en sousensembles dits " modules de base " qui correspondent à la structure du didacticiel.

La création préalable de ces tables offre un temps d'accès très court répondant à une interactivité " temps réel ".

Le logiciel, pour l'environnement Macintosh a été développé sous SuperCard 1,5 avec des extensions en langage C. La version compatible IBM (PS et AT) a été, quant à elle, écrite pour l'environnement graphique Windows 3 en langage $\mathrm{C}++$.

Le logiciel d'indexation/formatage des données et qui crée les tables interprétées par le logiciel de DIDACMER, est réalisé pour l'environnement Macintosh dans lequel les données ont été saisies. Pour la création des tables, ce logiciel interprète des codes régissant les liens entre les données ainsi que la présentation de ces données (couleur du texte, marge et tabulation, polices de caractère, insertion d'une image ou d'un graphique dans le texte).

\subsection{La saisie des données}

La saisie de toutes les données a été réalisée dans un environnement Apple. Les postes de travail, reliés en réseau Apple Talk, ont chacun pour élément de base un ordinateur Macintosh.

Couplés à certains de ces postes, on trouve le matériel suivant :

- un scanner couleur SHARP JX450 ;

- un scanner APPLE piloté par le logiciel de reconnaissance de caractère Omnipage ;

— une unité de sauvegarde magnéto-optique ;

- une imprimante laser.

Les logiciels utilisés pour la saisie, la gestion des données textuelles, et la numérisation du son, sont des " classiques » du marché et n'ont nécessité aucun développement d'interface logiciel spécifique pour la gestion des formats d'écriture/lecture.

Les fichiers de données multimédia sont identifiés par une codification de leur nom et leur position dans une arborescence de répertoires et sous-répertoires.

- Le nom indique :

- s'il s'agit d'un fichier lié au cours, ou aux exercices, ou à l'encyclopédie,

- à quel chapitre il se rapporte et sa numérotation dans ce chapitre.
- La place du fichier dans un sous-répertoire indique s'il s'agit d'un schéma, d'une photographie, d'une animation, d'un texte, de son, ou d'un fichier de présentation.

La procédure de saisie des données s'établie comme suit :

1. Saisie des textes (y compris une première relecture): - soit par traitement de texte ;

- soit par reconnaissance de caractère selon la qualité du document original.

2. Encodage des fichiers de texte. Les codes permettent :

- le découpage des données en "modules de base" (codes d'en-tête) servant à l'indexation et au chaînage de ces données;

- à chaque module de base est associé une liste de mots-clés ;

- l'insertion d'illustration et de formules dans le texte;

- la présentation en couleur du texte, la mise en page et le changement de police de caractères.

3. Réalisation des formules dans un logiciel d'écriture mathématique.

4. Digitalisation des photographies et de certains schémas.

5. Dessin de certains schémas dans un logiciel graphique de type vectoriel.

6. Reprise des schémas et formules pour la mise en couleur et des photographies pour y apporter les retouches finales dans un logiciel graphique de type point par point.

7. Création des animations.

8. Réalisation de la bande son.

9. Deuxième relecture (d'un point de vu plus scientifique) et corrections.

10. Indexation des données.

11. Relectures finales et vérification des liens.

12. Test auprès d'utilisateurs.

13. Mises au point définitives.

La mise en œuvre complète de DIDACMER et la planification des tâches se résume dans le graphe de la figure 12 .

\section{Suivi et mise à jour}

La réalisation d'une unité pédagogique multimédia sur disque CD-ROM comme DIDACMER a nécessité l'organisation d'un groupe de travail très structuré, ou chaque comité s'est engagé à réaliser une tâche précise.

Chaque individu est intégré dans une équipe de quelques personnes et a, à sa charge, une activité ponctuelle dans les domaines, technique, scientifique, informatique, pédagogique, commercial ou artistique.

L'organisation des groupes de travail se résume à l'organigramme de la figure 13. 


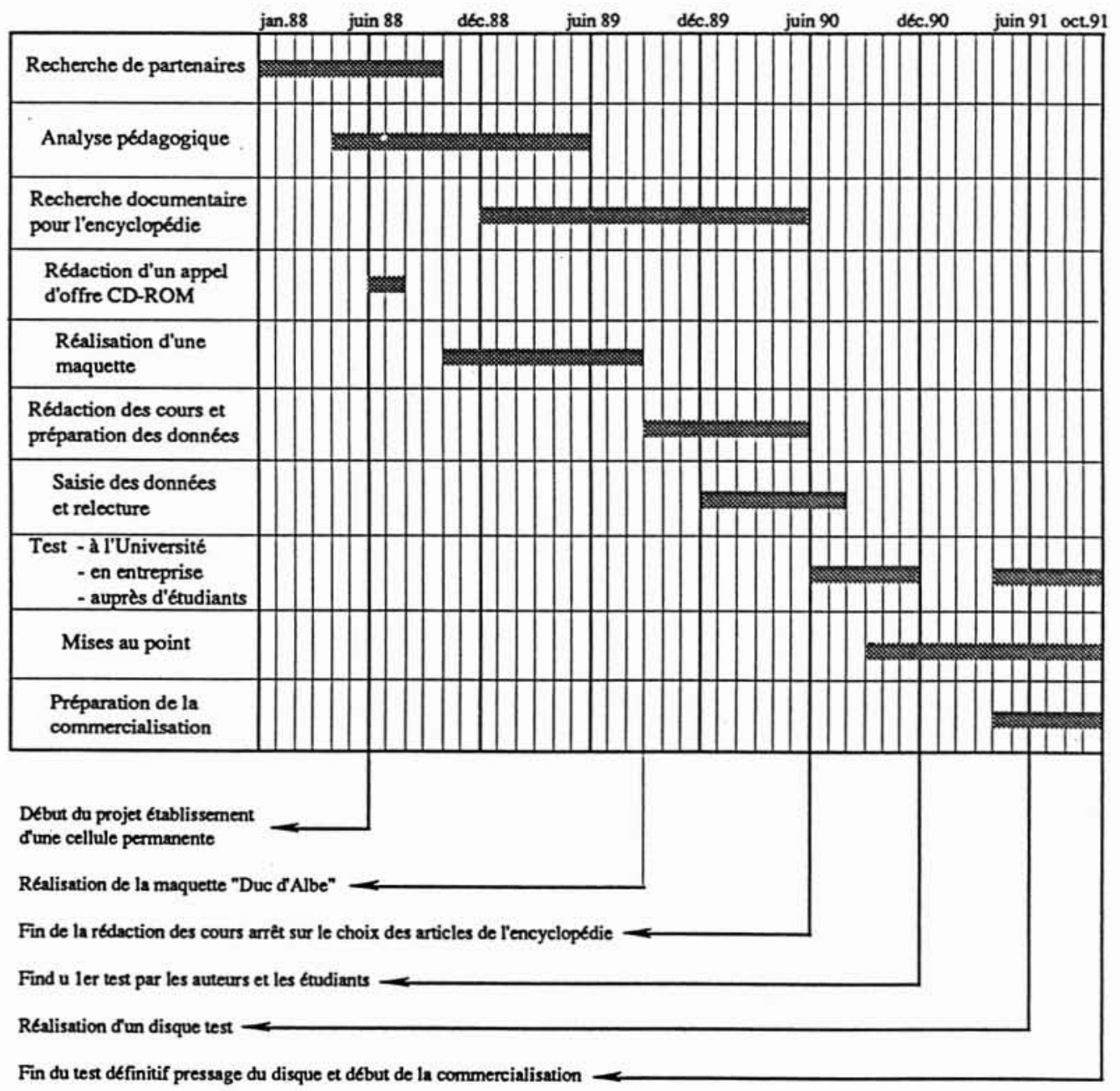

12. Planning de la réalisation de DIDACMER.

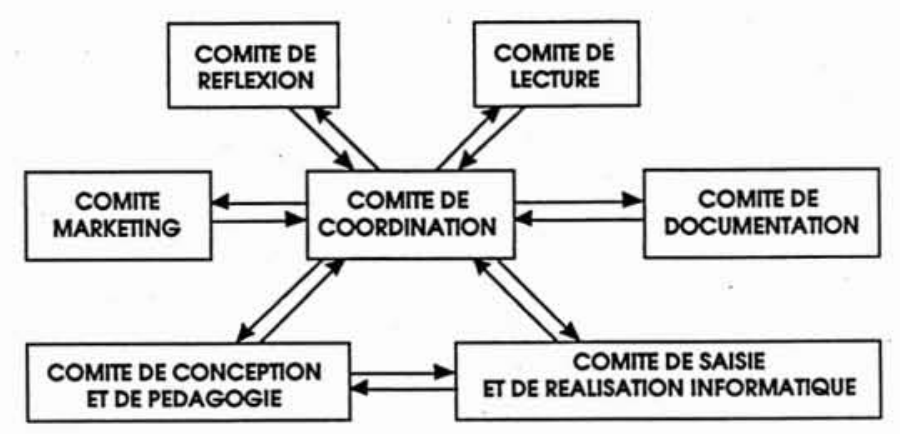

13. Organisation des groupes de travail pour la réalisation de DIDACMER. 
Le Comité de coordination est chargé des décisions à prendre quant aux démarches principales concernant la conception, la réalisation et la commercialisation du produit.

Il doit, entre autre, veiller à l'aboutissement du projet en coordonnant l'action des différentes équipes de travail.

Le Comité de réflexion doit créditer et attester de la qualité scientifique et du sérieux du travail réalisé. Son rôle est aussi de promouvoir et de valoriser le produit.

Le Comité de conception et de pédagogie est chargé de l'élaboration, de la réalisation et de la rédaction des articles indispensables au didacticiel. Il met aussi au point la démarche pédagogique à appliquer. Il est composé d'une équipe très importante d'ingénieurs, d'enseignants et de pédagogues spécialisés.

Le Comité de documentation est chargé de la recherche des articles nécessaires à la conception et à la réalisation du CD-ROM.

Le Comité de lecture intervient pour contrôler la qualité scientifique, technique et pédagogique des articles qui lui seront proposés. Ses membres sont sélectionnés pour leurs compétences et leur expérience.

Le Comité de saisie et de réalisation informatique est composé d'ingénieurs spécialistes des travaux maritimes et d'informaticiens qui supervisent la saisie de données dans une équipe familiarisée à l'utilisation de logiciels de traitement de texte et de logiciels graphiques.

Dans cette structure seul le comité de coordination dispose d'une cellule permanente composée d'un secrétariat et d'un ingénieur. C'est cette cellule qui organise désormais le suivi du projet.

Pour établir le contenu de la prochaine mise à jour (prévue pour décembre 1993) et assurer le suivi permanent du logiciel deux clubs ont été créés :

- un club composé des auteurs de DIDACMER,

- un club regroupant les utilisateurs.
Ces deux clubs sont intégrés à la cellule informatique du Centre français du littoral. Ainsi ils disposeront d'une rubrique dans le bulletin de liaison "Paralia " de cette association.

Les questions des utilisateurs, à propos du contenu de DIDACMER, transmises au secrétariat du Centre français du littoral, seront adressées à l'auteur concerné qui s'efforcera de répondre dans les meilleurs délais.

Les questions d'ordre informatique et concernant l'utilisation du logiciel ou de son installation seront adressées au secrétariat de la cellule permanente de DIDACMER.

Le contenu des mises à jour sera discuté au cours de réunions annuelles du club des auteurs et du club des utilisateurs auxquelles viendront se joindre les représentants de la cellule permanente de DIDACMER.

Les questions et les réponses paraîtront dans Paralia ainsi que le détail des modifications envisagées pour les prochaines mises à jour du logiciel et des données.

Par ailleurs, un autre élément moteur du suivi de DIDACMER est la mise en place du Diplôme d'Université de Travaux maritimes, délivré par l'Université de Nantes, et qui ponctuera la formation dispensée par DIDACMER. Ce diplôme, sous la responsabilité pédagogique d'Alain Grovel, Professeur à l'Université de Nantes, fonctionnera sous forme de tutorat et donnera lieu facultativement à des stages au sein de l'Université de Nantes. Ce diplôme pourra être mis en service dès la rentrée prochaine et l'expérience pédagogique qui en résultera permettra aussi l'amélioration du didacticiel.

Enfin, le contenu définitif de la mise à jour sera établi après réunion des comités tels qu'ils sont définis précédemment (fig. 13) et intègrera dans la mesure du possible les suggestions des clubs d'auteurs et d'utilisateurs ainsi que les remarques issues de l'expérience du Diplôme d'Université. Ces comités auront alors à charge la réalisation de la mise à jour.

\section{Conclusion}

Ainsi la réalisation de la première édition de DIDACMER n'est pas une fin en soit. C'est à l'usage que DIDACMER trouvera sa forme définitive et c'est avec la participation active de ces utilisateurs que DIDACMER collera à l'actualité.

Aussi, à terme, DIDACMER pourra devenir l'outil fédérateur du savoir-faire français scientifique et technique en matière de travaux maritimes.

La volonté du Service Formation continue de l'Université de Nantes est ainsi de poser les premiers jalons de l'Université ouverte, c'est-à-dire une université au sein même de votre domicile ou de votre lieu de travail, grâce à des outils pratiques et conviviaux qui se multiplieront et qui deviendront de moins en moins onéreux. 


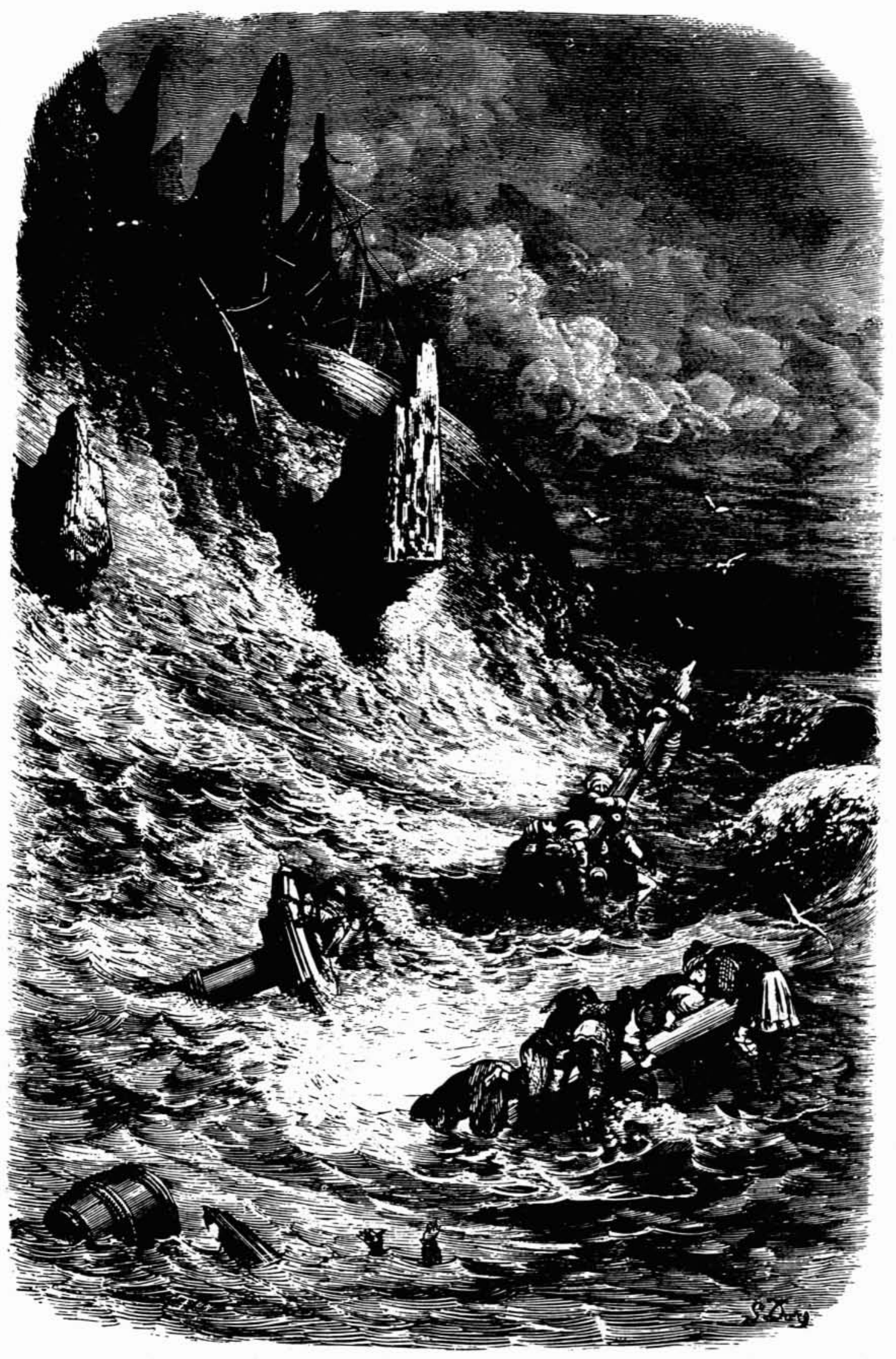

